\title{
Radiographic feasibility study of cortical bone trajectory and traditional pedicle screw dual trajectories
}

\author{
Jeffrey P. Mullin, MD, MBA, Breanna Perlmutter, BA, Eric Schmidt, MD, Edward Benzel, MD, and \\ Michael P. Steinmetz, MD
}

Department of Neurological Surgery, Cleveland Clinic Foundation, Cleveland, Ohio

\begin{abstract}
OBJECTIVE In 2009, Santoni and colleagues described a novel technique of posterior instrumentation; the cortical bone trajectory (CBT) was described as a caudocephalad and medial-to-lateral trajectory. Reported indications for CBT fixation include patients with osteoporosis, single-level degenerative disease, or adjacent-segment disease (ASD). In cases of revision surgery, it is technically possible and beneficial to place a traditional pedicle screw and a CBT screw at the same spinal level and side. It remains unclear as to the feasibility of placing both a traditional and a CBT screw at all levels of the lumbar spine and with varying trajectories of the preexisting traditional pedicle screws. Therefore, the authors conducted a study to radiographically assess the feasibility of using CBT and traditional pedicle screws at the same level in a large patient population.
\end{abstract}

METHODS Using a 3D Spine Navigation WorkStation, the authors assessed 47 lumbar spine CT scans. These images were obtained from 2 disparate groups of patients: those who had previously undergone traditional pedicle instrumentation (prior surgery group) and those who had not (no prior surgery group). The authors virtually placed traditional pedicle and CBT screws at each lumbar level bilaterally. It was then determined if the dual trajectories were feasible, as defined by the presence or absence of a collision of the screw trajectories based on 3D imaging.

RESULTS Overall, the authors evaluated 47 patients and were able to successfully plan dual trajectories in $50 \%$ of the pedicles. The no prior surgery group, compared with the prior surgery group, had a significantly greater success rate for dual trajectories. This difference was most significant in the lower lumbar levels $(L 3-5)$ where the prior instrumented group had success rates lower than $40 \%$ compared with the no prior surgery group's success rate, which was greater than $70 \%$. There was a significant difference between each lumbar level in the lower spine.

CONCLUSIONS There is a significant difference in the feasibility of planning CBT screws in patients who have undergone prior pedicle instrumentation compared with placing CBT and traditional pedicle screws simultaneously, but dual trajectory pedicle screws are a feasible option for posterior lumbar spinal instrumentation, especially as a de novo option in osteoporotic patients or in patients with ASD who underwent previous pedicle instrumentation. Ultimately, the practical clinical utility and biomechanical effects on the spine and instrumentation construct would require additional study.

http://thejns.org/doi/abs/10.3171/2016.4.SPINE151483

KEY WORDS pedicle instrumentation; cortical bone trajectory; lumbar fusion

$\mathrm{P}$ OSTERIOR spinal instrumentation has been used to achieve spinal fusion for decades. Currently, pedicle screw fixation using a "traditional" approach is the most common technique employed. This approach uses a trajectory that is along the axis of the pedicle with a cephalocaudad and lateral-to-medial trajectory. ${ }^{15,18}$ In 2009, Santoni et al. described a novel technique of posterior instrumentation; this cortical bone trajectory (CBT) was described as a caudocephalad and medial-to-lateral trajec- tory. CBT instrumentation has been reported to be beneficial in certain patient populations, including patients with osteoporosis, single-level degenerative disease, or adjacent-segment disease (ASD). ${ }^{12,14,15}$ Most studies describe CBT as a stand-alone posterior instrumentation option or in conjunction with an interbody device. Two studies exist in the literature that report clinical results of using CBT in conjunction with traditional pedicle screws (Fig. 1). Ueno and colleagues were the first to report the dual tra-

ABBREVATIONS ASD = adjacent-segment disease; $C B T=$ cortical bone trajectory.

SUBMITTED December 15, 2015. ACCEPTED April 26, 2016.

INCLUDE WHEN CITING Published online July 8, 2016; DOI: 10.3171/2016.4.SPINE151483. 


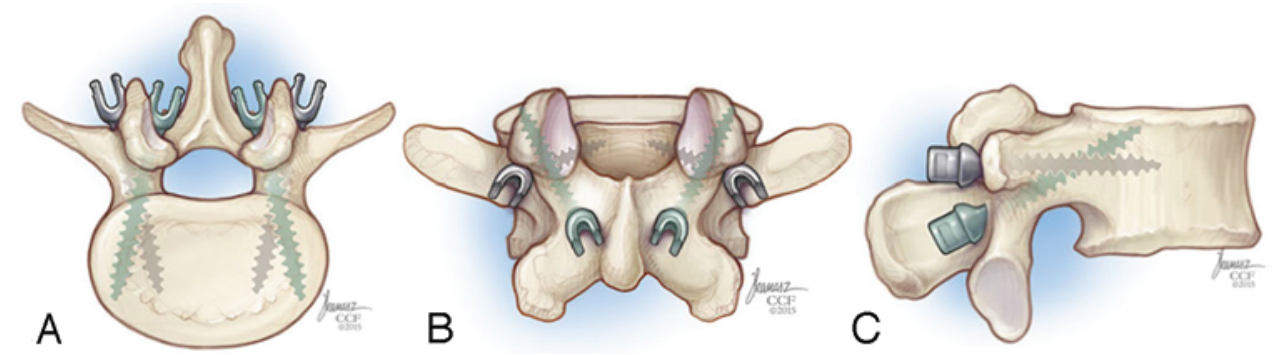

FIG. 1. Axial (A), posterior (B), and lateral (C) schematics demonstrating multiple screws in a single pedicle. Reprinted with permission, Cleveland Clinic Center for Medical Art \& Photography @ 2015. All Rights Reserved. Figure is available in color online only.

jectory technique in which CBT and traditional trajectory screws were inserted into the same pedicle in an attempt to "achieve the most solid fixation" in an osteoporotic woman with scoliosis; all screws were placed de novo. ${ }^{16}$ The additional screws and rods provided additional structural support and stability. In this patient, 2 rods were placed and connected to traditional pedicle screws and 2 additional rods were connected to the CBT screws (Fig. 2B). Hyun et al. demonstrated the added rigidity offered by using more rods and screws, in addition to the standard 2-rod constructs typically employed. ${ }^{3}$ The second case of dual trajectory screws was reported by Rodriguez et al. ${ }^{14}$ A particularly intriguing aspect of this report was their use of multiple pedicle screws in several pedicles. Of note, they reported placing CBT screws after traditional pedicle screws were placed in the same pedicle (Fig. 2C). In this scenario, the previously placed construct does not have to be exposed or connected to the new CBT construct at the additional level; this could result in decreased operative time and blood loss. To the best of our knowledge, these are the only studies that have made mention of the "dualtrajectory" technique. Our aim with this study was to conduct a radiographic study to determine the feasibility of using CBT and traditional pedicle screws at the same level in a larger population. Clearly, there could be biomechanical implications with the presence of additional hardware when using this dual trajectory technique, but we cannot address this issue without in vitro or in vivo studies. The intent of this study was to only evaluate radiographic feasibility.

\section{Methods}

The lumbar CT scans of 47 random patients were evaluated; the scans were the most recent studies at our institution that met the search criteria "pedicle screws" or "normal/no pathology" using our institutional radiographic database. The images were divided into 2 groups: 24 patients who had preexisting traditional pedicle screws (prior surgery group: age range $44-85$ years, mean 58.7 \pm 12.5 years $[ \pm \mathrm{SD}] ; 17$ females) and 23 patients who had no previous instrumentation (no prior surgery group: age range $24-81$ years; mean $58.3 \pm 12.4$ years; 10 females). The CT scans were analyzed using 3D reconstruction software (Brainlab Elements DICOM Viewer, Brainlab).

\section{Digital Placement of Traditional Pedicle Screws}

For those patients in the prior surgery group, digital pedicle screws were added to precisely define the locations of the screws already in place. Next, $6.5-\mathrm{mm}$ screws were placed in the remaining lumbar pedicles using the lateral-to-medial and inferior trajectory along the axis of the pedicle as previously described (Fig. 1). ${ }^{15,18}$ For those patients with no prior instrumentation, $6.5-\mathrm{mm}$ pedicle
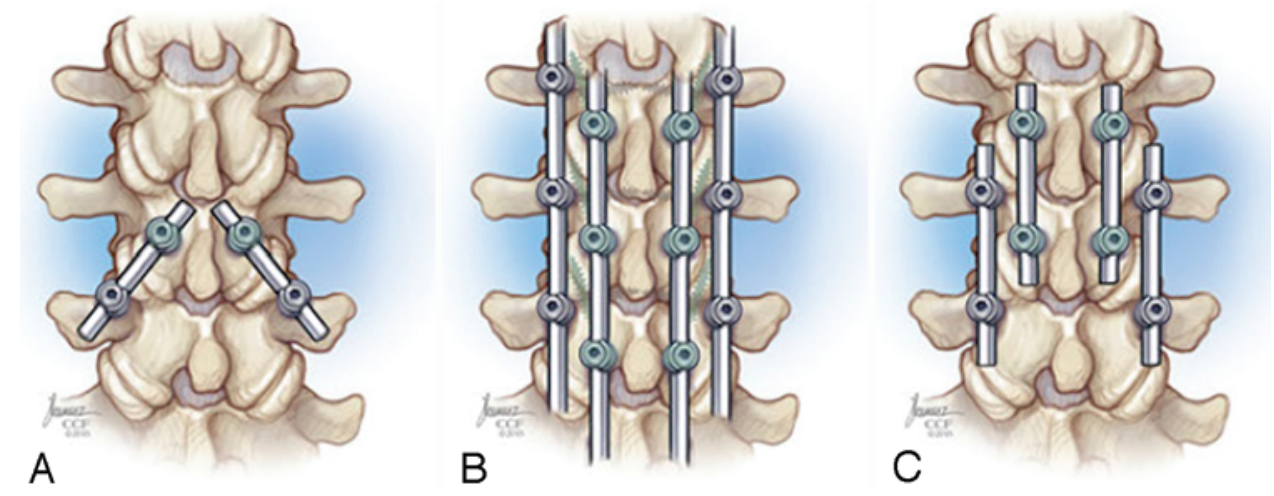

FIG. 2. Clinical scenarios for hybrid CBT-traditional pedicle screw instrumentation. A: Single-level fusion with CBT screws placed superiorly and traditional pedicle screws placed caudally. B: Four-column fusion with parallel CBT rods and traditional rods. C: Superior adjacent-level disease treated with freestanding single-level CBT fusion in addition to prior traditional rod construct. Reprinted with permission, Cleveland Clinic Center for Medical Art \& Photography @ 2015. All Rights Reserved. Figure is available in color online only. 
screws were virtually placed in all 10 pedicles in the same manner as for those patients in the previous instrumentation group (Fig. 3).

\section{Digital Placement of CBT Screws}

The entry point of the CBT screws was the rostrocaudal midpoint of the pars interarticularis. A medial-to-lateral and superior intrapedicular trajectory was then followed, as described previously. ${ }^{2}$ Both $4.5-$ and $4.0-\mathrm{mm}$ screws were virtually inserted into each pedicle; this smaller diameter is within standard range for the CBT. ${ }^{12,13,16,17}$ For those patients with prior fusion, most CBT screws passed caudal to the preexisting traditional pedicle screws. However, in several patients, placement of a CBT screw was only feasible when adjusting the entry point rostrally to allow the CBT screw to pass rostral to the preexisting traditional pedicle screw. For patients in both groups, it was then determined if the trajectories of the traditional pedicle screws and the CBT screws (4.5- and 4.0-mm screws considered separately) collided within the pedicle or vertebral body at each level.

\section{Statistical Analysis}

Dual trajectory planning was considered successful if the trajectories of the traditional pedicle screw and the CBT screw did not collide. In the prior surgery group, the percentages of successfully planned dual trajectories were then calculated for each lumbar level and for each size of CBT screw. A 2-tailed t-test was then used to compare the success of planned dual trajectories across multiple lumbar levels. This analysis was repeated for those patients with no prior instrumentation (no prior surgery group). Additionally, a Fisher exact test was used to analyze the percent success rate at each lumbar level between the group of patients with prior fusion and those without prior fusion.

\section{Results}

Overall, 47 patients underwent lumbar CT scanning. A total of 222 pedicle screws had been placed in the patients who previously underwent instrumentation. The fused level was not spread evenly across the various levels. Only 3 patients had previous L-1 pedicle screws placed, compared with 20 patients who had L-5 screws placed.

There were a limited number of patients who underwent previous pedicle instrumentation in the upper lumbar spine. Only 6 pedicle screws had been placed at the L-1 vertebral level and only 12 had been placed at the L-2 level. Because of this limited sample size, significance between the prior surgery and the no prior surgery groups could not be determined. However, for the L-1 levels in the no prior surgery group, we found that we were able to successfully place a CBT and traditional pedicle screw $35.2 \%$ and $34.1 \%$ of the time when using $4.0-$ or $4.5-\mathrm{mm}$ CBT screws, respectively.

For the lower lumbar levels, we were able to find significant differences between the previously fused group and the virgin group at all levels. Overall, we were able to successfully plan dual trajectory screws $38 \%$ of the time when previous pedicle screws had been placed. In the patients in whom we planned both trajectories, we were able to successfully plan for dual trajectories in $70 \%$ of the pedicles $(\mathrm{p}<0.005)$.

When examining each of the lower lumbar levels individually, we again found a significant difference between the prior surgery and no prior surgery groups (Table 1). At L-3, the CBT-traditional pedicle dual trajectory was successfully planned $20 \%$ of the time with both $4.0-$ and 4.5-mm CBT screws in those patients with prior surgery, compared with $50 \%(\mathrm{p}=0.022)$ and $48.6 \%(\mathrm{p}=0.024)$ for 4.0- and 4.5-mm CBT screws, respectively, in those with no prior surgery.

Similar differences were found at L-4 and L-5. At L-4
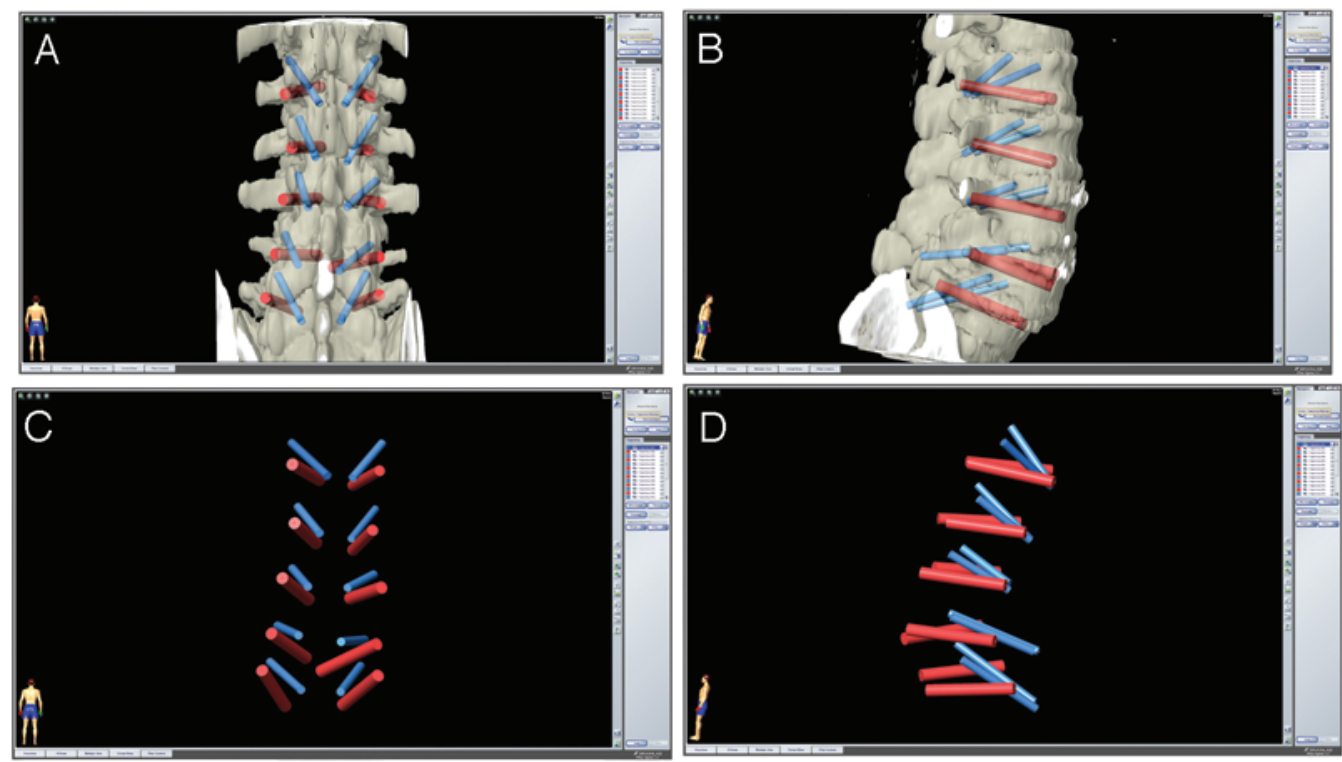

FIG. 3. Brainlab planning overview showing the posterior view (A), lateral view (B), posterior view (screws only) (C), and lateral view (screws only) (D). Figure is available in color online only. 
TABLE 1. Results of radiographic planning dual trajectory screw placement

\begin{tabular}{|c|c|c|c|c|c|c|c|c|c|}
\hline \multirow{3}{*}{$\begin{array}{c}\text { Spine Level/ } \\
\text { Screw Size (mm) }\end{array}$} & \multicolumn{8}{|c|}{ Dual Trajectory Successfully Planned? } & \multirow[b]{3}{*}{$p$ Value $^{*}$} \\
\hline & \multicolumn{4}{|c|}{ Prior Fusion Group } & \multicolumn{4}{|c|}{ No Prior Fusion Group } & \\
\hline & No & Yes & Total Pedicles & \% Successfully Planned & No & Yes & Total Pedicles & $\%$ Successfully Planned & \\
\hline$L-1 / 4.0$ & 3 & 3 & 6 & 50 & 57 & 31 & 88 & 35.2 & 0.664 \\
\hline$L-1 / 4.5$ & 3 & 3 & 6 & 50 & 58 & 30 & 88 & 34.1 & 0.662 \\
\hline $\mathrm{L}-2 / 4.0$ & 4 & 8 & 12 & 66.7 & 60 & 22 & 82 & 26.8 & 0.017 \\
\hline $\mathrm{L}-2 / 4.5$ & 5 & 7 & 12 & 58.3 & 62 & 20 & 82 & 24.4 & 0.036 \\
\hline$L-3 / 4.0$ & 16 & 4 & 20 & 20 & 37 & 37 & 74 & 50 & 0.022 \\
\hline L-3/4.5 & 16 & 4 & 20 & 20 & 38 & 36 & 74 & 48.6 & 0.024 \\
\hline L-4/4.0 & 20 & 13 & 33 & 39.4 & 9 & 52 & 61 & 85.2 & 0.001 \\
\hline L-4/4.5 & 20 & 13 & 33 & 39.4 & 14 & 47 & 61 & 77 & 0.001 \\
\hline L-5/4.0 & 19 & 21 & 40 & 52.5 & 11 & 43 & 54 & 79.6 & 0.001 \\
\hline L-5/4.5 & 19 & 21 & 40 & 52.5 & 11 & 43 & 54 & 79.6 & 0.001 \\
\hline
\end{tabular}

* Fisher exact test.

in the prior surgery group, the dual trajectory planning was successful $39.9 \%$ of the time for both 4.0- and 4.5$\mathrm{mm}$ CBT screws. When evaluating the no prior surgery group at L-4, we were able to successfully plan the dual trajectory $85.2 \%(p=0.001)$ of the time with $4.0-\mathrm{mm}$ CBT screws and $77.0 \%(p=0.001)$ of the time with the $4.5-\mathrm{mm}$ CBT screws. At L-5 in the prior surgery group, the dual trajectory planning was successful $52.5 \%$ of the time for both 4.0- and 4.5-mm CBT screws. When evaluating the no prior surgery group at L-5, we were successfully able to plan the dual trajectory $79.6 \%(\mathrm{p}=0.001)$ of the time with both 4.0 - and 4.5 -mm CBT screws.

\section{Discussion}

The mainstay of posterior spinal instrumentation remains traditional pedicle screws; however, recent developments have led to an increased interest in the CBT technique. Following Santoni and colleagues' initial publication, ${ }^{15}$ Mobbs further defined the cortical bone trajectory, describing it as the "medio-latero-superior trajectory," using an entry point at the lateral pars. ${ }^{12}$ Mobbs also discussed the use of hybrid CBT and traditional pedicle screw instrumentation for single-level fusion (Fig. 2A). Matsukawa et al. reported on radiographic studies in which they evaluated the morphometric measurements pertaining to CBT throughout the spine. ${ }^{7,911}$ It was theorized that the CBT technique would result in "greater holding screw strength" due to the increased screw-cortical bone interface. ${ }^{5,15}$ In vitro studies have demonstrated that the pullout and toggle characteristics of CBT are at least equivalent to those of traditional trajectory pedicle screws. ${ }^{4,13,15}$ Biomechanical concerns have been raised by some regarding the use of CBT screws. Depending on the length of the screw, they may not pass the instantaneous axis of rotation or, at minimum, reach it. This may limit the biomechanics of the construct, which then likely mandates the use of an interbody graft. The biomechanics were evaluated by Perez-Orribo et al. and demonstrate sound fixation when comparing CBT screws with interbody fusion to traditional pedicle screws with interbody fusion..$^{13}$ CBT screws may, in fact, be biomechanically superior to traditional pedicle screws. ${ }^{1,8,17}$ An in vivo study demonstrated that the insertional torque was 1.7 times greater with CBT screws than with traditional pedicle screws..$^{10}$ It has been shown that insertional torque directly correlates with pullout strength, ${ }^{19}$ hence the significance of this study. These biomechanical studies were all conducted in vitro; long-term clinical studies are needed to evaluate not only immediate biomechanical outcomes but also long-term fusion rates. It is uncertain if the fusion rates resulting from this technique will improve due to increased rigidity of the instrumentation or worsen due to the decrease in the bone surface area of the construct. Furthermore, biomechanical studies have shown that CBT can be used as a rescue option when traditional pedicle screws fail. ${ }^{2}$ In addition, some have advocated the use of CBT screws for osteoporotic patients due to increased screw-bone interface integrity related to cortical bone purchase, while being a less invasive option for either de novo or ASD patients. ${ }^{14-16}$ When used for ASD, the CBT technique offers the benefit of not requiring the removal of previously placed instrumentation, thus significantly reducing the operative exposure required.${ }^{16}$ Whether for providing increased construct strength or attempting to limit surgical exposure, using the dual trajectory approach may become a valid option in appropriate patients.

To the best of our knowledge, there are 2 reports in the literature describing the dual trajectory technique..$^{14,16}$ Neither of these papers report issues associated with placing both screws in the same pedicle, although Ueno et al. mentioned the possible complications associated with the dual trajectory placement: “...there is a possibility of the surgeon being unable to insert TT [traditional trajectory] screws due to interference with screws in the pedicle; fracture of the pedicle may occur; or a screw may go beyond the lower wall of the pedicle and damage the root." ${ }^{\prime \prime}$ Ueno et al. and Rodriguez et al. did mention the need to obtain preoperative CT studies to aid in surgical planning..$^{14,16}$ Rodriguez et al. commented on this step, which allows one to evaluate "pedicle size, along with the pedicular placement of the existing screw [which] is a critical determinant of whether a second screw may be added safely." ${ }^{14}$ It can be assumed that their $100 \%$ success rate with the dual trajectory screw placement is related, in 
part, to careful patient screening. The only literature reports of CBT placement errors are from Santoni and colleagues' original description of the technique and Ueno and colleagues' technical note. ${ }^{5,16}$ Santoni et al. reported that $20 \%$ of the CBT screws resulted in a medial wall breach. ${ }^{15}$ Ueno et al. were unable to place the CBT in one pedicle due to the entry point fracturing after inserting the screw. ${ }^{16}$

The relative paucity of data regarding the feasibility of the dual trajectory technique led us to perform a radiographic analysis of a larger population. Regardless of prior fusion status, we were able to successfully plan dual trajectories in roughly half of the pedicles. There is an obvious trend to higher success rates in the lower lumbar region. This is to be expected, given that the pedicle significantly increases in size from rostral to caudal. ${ }^{11}$ While this information is useful to help clinicians consider the possibility of placing CBT screws in conjunction with traditional pedicle screws, it does not negate the need to obtain preoperative CT scans for feasibility planning.

While we demonstrated that the dual trajectory technique is feasible in a large percentage of the population, the significant difference between planning of the dual trajectory technique de novo is far superior to planning CBT screw placement after traditional pedicle screws have been placed without consideration for making room for the CBT screws. When planning de novo, the alteration of the traditional pedicle screw trajectory to accommodate subsequent placement of CBT screws makes a significant difference. Additionally, while we had no issue with radiographically planning CBT for patients who had undergone previous lumbar decompression, in actuality surgeons may have a more difficult time exposing the medial pars entry point needed for the CBT due to postoperative changes. Typically, the successful cases had traditional pedicle screws aimed more caudal and with lower entry points (Fig. 2). This results in CBT screws crossing rostral to the traditional screws, as was the case for Ueno et al. and Rodriguez et al. ${ }^{14,16}$ Interestingly, there were a few traditional screw placements that would allow for a CBT screw to be planned crossing caudal to the previously placed screw (Fig. 4), although this may be biomechanically suboptimal compared with the CBT crossing rostrally. ${ }^{6}$

While we believe that this report meaningfully adds to the literature, it is not without limitations. Most notably, this study was limited to radiographic analysis only. While we were able to clearly show a large portion of patients are able to have dual trajectory screws placed, we are unable to comment on the clinical effect of actually inserting these screws. Cadaveric and biomechanical studies are still needed. Additionally, another limitation was the large number of females in the sample size, especially in the fusion group. This may have led to a slightly lower success rate due to the typically smaller pedicle size in women. For our study, we used $6.5-\mathrm{mm}$ traditional pedicle screws at each level and 4.0- and 4.5-mm CBT screws. These values differ slightly from those in other reports. For example, Rodriguez et al. reported using at least 5.5$\mathrm{mm}$ CBT screws and 7.0-mm traditional pedicle screws. This difference may have led to over-reporting the success rate in our study, in which we used smaller screws. ${ }^{14}$ Con-

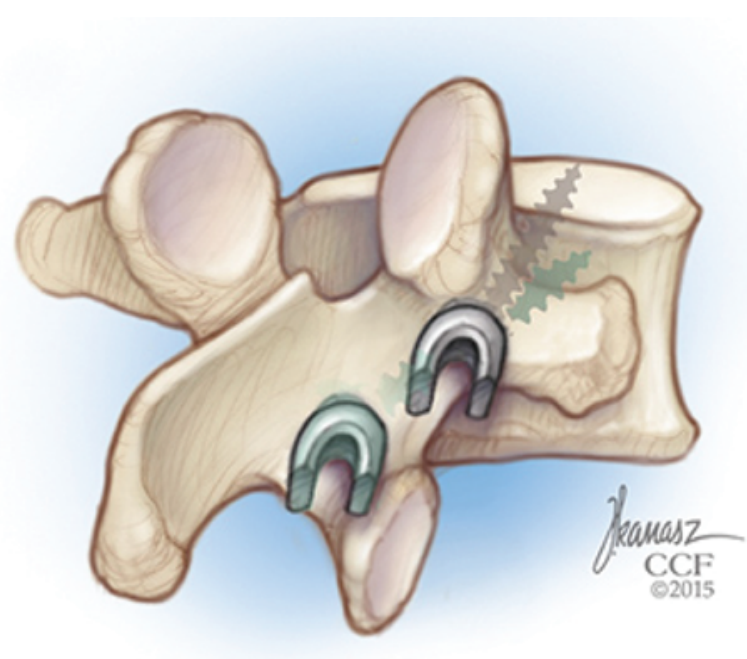

FIG. 4. Alternative dual pedicle trajectory with CBT crossing inferiorly and lateral to traditional pedicle screw. Reprinted with permission, Cleveland Clinic Center for Medical Art \& Photography @ 2015 . All Rights Reserved. Figure is available in color online only.

versely, Ueno et al. used screw sizes of $4.5 \mathrm{~mm}$ for CBT and $5.5 \mathrm{~mm}$ for traditional pedicle screws. ${ }^{16}$

\section{Conclusions}

There is a significant difference in the feasibility of planning CBT screws in patients with prior pedicle instrumentation compared with placing CBT and traditional pedicle screws simultaneously. However, dual trajectory pedicle screws are a feasible option for posterior lumbar spinal instrumentation, especially as a de novo option in osteoporotic patients or in patients with ASD who underwent previous pedicle instrumentation. Ultimately, the practical clinical utility and biomechanical effects on the spine and instrumentation construct require additional study.

\section{Acknowledgments}

A special thank you to Kris Dorn, radiology technician, and Don Leblanc for their help with the neuronavigation imaging system.

\section{References}

1. Baluch DA, Patel AA, Lullo B, Havey RM, Voronov LI, Nguyen NL, et al: Effect of physiological loads on cortical and traditional pedicle screw fixation. Spine (Phila Pa 1976) 39:E1297-E1302, 2014

2. Calvert GC, Lawrence BD, Abtahi AM, Bachus KN, Brodke DS: Cortical screws used to rescue failed lumbar pedicle screw construct: a biomechanical analysis. J Neurosurg Spine 22:166-172, 2015

3. Hyun SJ, Lenke LG, Kim YC, Koester LA, Blanke KM: Comparison of standard 2-rod constructs to multiple-rod constructs for fixation across 3-column spinal osteotomies. Spine (Phila Pa 1976) 39:1899-1904, 2014

4. Inceoğlu S, Montgomery WH Jr, St Clair S, McLain RF: Pedicle screw insertion angle and pullout strength: comparison of 2 proposed strategies. J Neurosurg Spine 14:670676, 2011

5. Kojima K, Asamoto S, Kobayashi Y, Ishikawa M, Fukui Y: 
Cortical bone trajectory and traditional trajectory-a radiological evaluation of screw-bone contact. Acta Neurochir (Wien) 157:1173-1178, 2015

6. Matsukawa K, Taguchi E, Yato Y, Imabayashi H, Hosogane $\mathrm{N}$, Asazuma T, et al: Evaluation of the fixation strength of pedicle screws using cortical bone trajectory: What is the ideal trajectory for optimal fixation? Spine (Phila Pa 1976) 40:E873-E878, 2015

7. Matsukawa K, Yato Y, Hynes RA, Imabayashi H, Hosogane $\mathrm{N}$, Asazuma T, et al: Cortical bone trajectory for thoracic pedicle screws: A technical note. J Spinal Disord Tech [epub ahead of print], 2014

8. Matsukawa K, Yato Y, Imabayashi H, Hosogane N, Asazuma T, Nemoto K: Biomechanical evaluation of the fixation strength of lumbar pedicle screws using cortical bone trajectory: a finite element study. J Neurosurg Spine 23:471-478, 2015

9. Matsukawa K, Yato Y, Kato T, Imabayashi H, Asazuma T, Nemoto K: Cortical bone trajectory for lumbosacral fixation: penetrating S-1 endplate screw technique: technical note. J Neurosurg Spine 21:203-209, 2014

10. Matsukawa K, Yato Y, Kato T, Imabayashi H, Asazuma T, Nemoto K: In vivo analysis of insertional torque during pedicle screwing using cortical bone trajectory technique. Spine (Phila Pa 1976) 39:E240-E245, 2014

11. Matsukawa K, Yato Y, Nemoto O, Imabayashi H, Asazuma T, Nemoto K: Morphometric measurement of cortical bone trajectory for lumbar pedicle screw insertion using computed tomography. J Spinal Disord Tech 26:E248-E253, 2013

12. Mobbs RJ: The "medio-latero-superior trajectory technique": an alternative cortical trajectory for pedicle fixation. Orthop Surg 5:56-59, 2013

13. Perez-Orribo L, Kalb S, Reyes PM, Chang SW, Crawford NR: Biomechanics of lumbar cortical screw-rod fixation versus pedicle screw-rod fixation with and without interbody support. Spine (Phila Pa 1976) 38:635-641, 2013

14. Rodriguez A, Neal MT, Liu A, Somasundaram A, Hsu W, Branch CL Jr: Novel placement of cortical bone trajectory screws in previously instrumented pedicles for adjacentsegment lumbar disease using CT image-guided navigation. Neurosurg Focus 36(3):E9, 2014

15. Santoni BG, Hynes RA, McGilvray KC, Rodriguez-Canessa
G, Lyons AS, Henson MA, et al: Cortical bone trajectory for lumbar pedicle screws. Spine J 9:366-373, 2009

16. Ueno M, Imura T, Inoue G, Takaso M: Posterior corrective fusion using a double-trajectory technique (cortical bone trajectory combined with traditional trajectory) for degenerative lumbar scoliosis with osteoporosis: technical note. J Neurosurg Spine 19:600-607, 2013

17. Ueno M, Sakai R, Tanaka K, Inoue G, Uchida K, Imura T, et al: Should we use cortical bone screws for cortical bone trajectory? J Neurosurg Spine 22:416-421, 2015

18. Weinstein JN, Spratt KF, Spengler D, Brick C, Reid S: Spinal pedicle fixation: reliability and validity of roentgenogram-based assessment and surgical factors on successful screw placement. Spine (Phila Pa 1976) 13:1012-1018, 1988

19. Zdeblick TA, Kunz DN, Cooke ME, McCabe R: Pedicle screw pullout strength. Correlation with insertional torque. Spine (Phila Pa 1976) 18:1673-1676, 1993

\section{Disclosures}

The authors report no conflict of interest concerning the materials or methods used in this study or the findings specified in this paper.

\section{Author Contributions}

Conception and design: Mullin. Acquisition of data: Mullin, Perlmutter, Schmidt. Analysis and interpretation of data: Mullin. Drafting the article: Mullin, Perlmutter. Critically revising the article: Benzel. Reviewed submitted version of manuscript: all authors. Approved the final version of the manuscript on behalf of all authors: Mullin. Statistical analysis: Mullin. Study supervision: Benzel, Steinmetz.

\section{Correspondence}

Jeffrey Mullin, Department of Neurological Surgery, Cleveland Clinic Foundation, 9500 Euclid Ave., Mail Code S100, Cleveland, OH 44195. email: mullinj5@ccf.org. 